
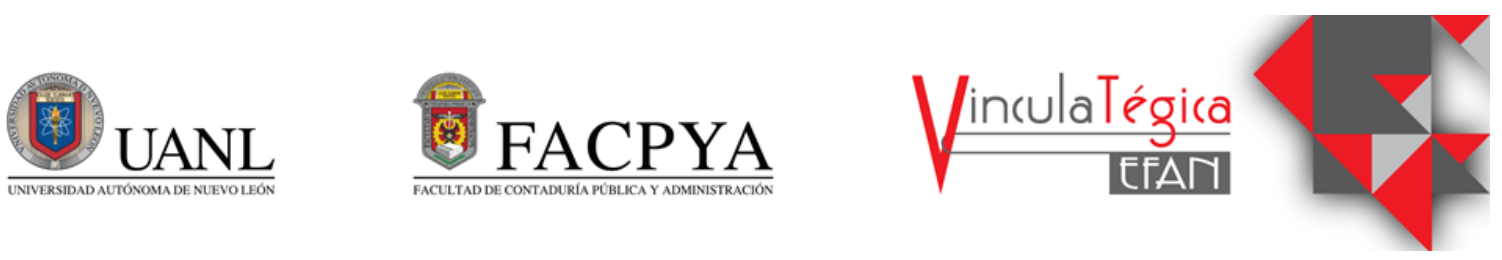

\title{
Diseño de un instrumento que mide las dimensiones del clima organizacional en una empresa maquiladora en Mexicali (B.C.)
}

\author{
Claudia Gabriela Sandoval Silva ${ }^{1}$, Loreto María Bravo Zanoguera ${ }^{2}$ y Gloria Muñoz del Real ${ }^{3}$ \\ 'Universidad Autónoma de Baja California, Facultad de Ciencias Administrativas, \\ claudia.sandoval97@uabc.edu.mx, Mexicali,B.C., (+52) 6862302371. \\ ${ }_{2}^{2}$ Universidad Autónoma de Baja California, Facultad de Ciencias Administrativas, \\ loreto@uabc.edu.mx, Mexicali, B.C., (+52) 6861094916. \\ ${ }^{3}$ Universidad Autónoma de Baja California, Facultad de Ciencias Administrativas, \\ gloria.munoz@uabc.edu.mx, Mexicali,B.C., (+52)6861199350.
}

Información del artículo revisado por pares

Fecha de aceptación: junio-2021

Fecha de publicación en línea: diciembre-2021

DOI: https://doi.org/10.29105/vtga7.2-33

\section{Resumen}

En la actualidad el clima organizacional de una organización y el factor humano es determinante para la obtención y logro de los objetivos que cada empresa va determinando a lo largo del tiempo, y es de suma importan para el desarrollo de estrategias para poder visualizar el futuro. El principal objetivo de este trabajo es la validación de un instrumento que identifique cual dimensión del clima organizacional presente mayor influencia sobre la productividad en el área de finanzas de una maquiladora ubicada en Mexicali, B.C. El instrumento consistió en un cuestionario de 29 ítems del cual se incluyeron las dimensiones de liderazgo, motivación, reciprocidad y participación las cuales fueron validadas por diversas pruebas estadísticas entre ellas KMO, Alfa de Cronbach entre otras, todas en el en el programa SPSS. Como instrumento final quedaron únicamente 26 ítems, de los cuales la dimensión de reciprocidad fue la que obtuvo mejores índices de las pruebas realizadas siendo el ítem RR19 el que mayor correlación tiene respecto a otros ítems.

Palabras clave: Liderazgo, motivación, reciprocidad, participación.

\section{INTRODUCCIÓN}

El clima organizacional hoy en día es un tema relevante para la adaptación de los empleados

\begin{abstract}
Currently the organizational climate of an organization and the human factor is decisive for obtaining and achieving the objectives that each company determines over time, and it is extremely important for the development of strategies to be able to visualize the future. The main objective of this work is the validation of an instrument that identifies which dimension of the organizational climate has the greatest influence on productivity in the finance area of a maquiladora located in Mexicali, B.C. The instrument consisted of a 29-item questionnaire which included the dimensions of leadership, motivation, reciprocity, and participation, which were validated by various statistical tests, including KMO, Cronbach's Alpha, among others, all in the SPSS program. As a final instrument, only 26 items remained, of which the reciprocity dimension was the one that obtained the best rates of the tests performed, with the RR19 item being the one with the highest correlation with other items.
\end{abstract}

Keywords: Leadership, motivation, reciprocity, participation.

JEL: M12, M19, M54.

dentro de una organización, ya que se considera que es uno de los factores más importantes para la obtención y logro oportuno de los objetivos. 
Luthans (2008), define el clima organizacional como "el estudio y la aplicación de las fortalezas y las capacidades psicológicas positivas de los recursos humanos, que se miden, se desarrollan y se administran para el mejoramiento de la productividad en el lugar del trabajo actual. Salazar, Guerrero y Cañedo (2009), mencionan que un clima laboral positivo favorece en el cumplimiento de metas y objetivos porque da un sentido de pertenencia a los empleados. Destacando a su vez que un clima negativo, se presenta principalmente por la falta de identidad en metas y objetivos, lo cual constituye un deterioro del ambiente de trabajo ocasionando rendimiento en su productividad.

El presente trabajo se realizó en una empresa maquiladora, dedicada a la fabricación de microcircuitos en Mexicali, B.C, específicamente en el área de finanzas la cual está integrada por tres departamentos costos, contabilidad y nómina que cuentan con un total de 30 empleados, más 3 gerentes, 1 supervisor y un contralor, actualmente en esta área se presenta una disminución de la productividad. Esta disminución trae como consecuencia un mal desempeño laboral, el incumplimiento de objetivos y metas, retraso en los tiempos de entrega de las actividades, conflictos gerenteempleado, que repercuten en la comunicación, confianza y clima organizacional. Las actividades, así como los roles se encuentran muy bien definidos para cada uno de los empleados, para la empresa los colaboradores son parte.

\section{MARCO TEÓRICO}

En este apartado se abordan las teorías utilizadas para la operacionalización de las variables.

\section{Clima organizacional}

El clima organizacional se arraiga en las percepciones individuales, y se define como aquellos patrones repetitivos de comportamiento, sentimientos y actitudes que caracterizan la vida en la organización, relacionan las situaciones actuales dentro de una organización y los vínculos que existen entre los equipos de trabajo, los empleados y el desempeño laboral. (Ramírez y Domínguez 2012).

El clima en las empresas está integrado principalmente por elementos como: fundamental en logro de los objetivos y metas, por lo que siempre busca su motivación y desarrollo. El clima organizacional debe medirse ya que no basta con suponer conclusiones sin sustento, es necesario conocer las causas y motivos del desempeño de los empleados y comprender la relación del clima organizacional en la productividad del área de finanzas.

\section{Pregunta de investigación}

¿Cómo se puede determinar la dimensión del clima organizacional con mayor influencia sobre la productividad del área de finanzas?

\section{Hipótesis}

H1. La aplicación de un instrumento de medición puede detectar que dimensión del clima organizacional influye mayormente en el incremento de la productividad en el área de finanzas.

H2. La aplicación de un instrumento de medición no determina que dimensión del clima organizacional influye mayormente en el incremento de la productividad en el área de finanzas.

\section{Objetivo}

El objetivo del presente trabajo consiste en validar un instrumento que permita identificar que dimensión del clima organizacional tiene mayor influencia sobre la productividad dentro del área de finanzas.

a) Aspectos individuales de los empleados en el que se consideran las percepciones, actitudes, valores, aprendizaje y estrés que pueden sentir los empleados.

b) Los grupos dentro de la organización, su estructura.

c) La motivación y necesidades.

d) Liderazgo, políticas, influencia, estilo.

e) La estructura con su macro y micro dimensiones.

f) Los procesos organizacionales, evaluación, sistema de remuneración, comunicación y el proceso de toma de decisiones.

\section{Dimensiones del Clima Organizacional}

Recio, Balderas y Martínez (2016) mencionan el modelo de análisis de clima organizacional, propuesto por la Organización Panamericana de 
la Salud (OPS) donde define en cuatro grandes dimensiones: Liderazgo, Motivación, Reciprocidad y Participación.

\section{Liderazgo}

Según Marín, Melgar y Castaño (1992) definen el liderazgo como la "Influencia que ejerce un individuo en el comportamiento de otras personas en la búsqueda, eficiente y eficaz, de objetivos previamente determinados, por medio de la habilidad de orientar y convencer a otras para ejecutar, con entusiasmo las actividades asignadas". "El liderazgo es el proceso de influencia entre líderes y seguidores para lograr los objetivos organizacionales por medio del cambio" Lussier y Achua (2011).

Indicadores del Liderazgo

- Dirección: Proporciona el sentido de orientación de las actividades de una unidad de trabajo, estableciendo los objetivos e identificando las metas que se deben alcanzar y los medios para lograrlo.

- $\quad$ Estímulo del trabajo en equipo: Busca el logro de objetivos comunes. Conocer y compartir hacia donde se quiere llegar.

\section{Motivación}

Cubas (2016), citando a Tejedor (2014), indica que la motivación, es el comportamiento que presenta una persona cuando decide alcanzar una meta, es decir, los colaboradores que están motivados tendrán un nivel alto de desempeño y tratarán de lograr a toda costa sus objetivos, dado que las metas fijadas por las organizaciones, las toman como propias.

\section{La motivación de los empleados mediante las} expectativas del desempeño

Slocum (2009) citado por Sum (2015), define parte de la motivación del desempeño es importante crear empleos que resulten retadores y gratificantes para las personas, así como conocer que si se esfuerzan por alcanzar sus metas y objetivos tendrás las recompensas esperadas.

De acuerdo con el modelo de las expectativas, las personas se sienten motivadas si piensan que el trabajo les va a poder proporcionar cosas que necesitan y desean. El objetivo de este modelo es que sostiene que si la persona tiene necesidades e ideas propias respecto a lo que desea de su trabajo en el momento de tomar una decisión respecto a cualquier asunto o tema o con el hecho de saber respecto a que organización entrara el individuo y cuan duro será su trabajo actuara basado en sus necesidades e ideas.

Indicadores de Motivación:

- Realización Personal: Contexto ocupacional en el cual aplica sus habilidades

- Reconocimiento de la aportación: Cuando la organización reconoce y da crédito al esfuerzo realizado por cada persona, en la ejecución de las tareas asignadas para el logro de los objetivos.

- Adecuación de las condiciones de trabajo: Las condiciones físicas, ambientales y psicosociales, en que se realiza el trabajo diario. Marín, Melgar y Castaño (1992).

\section{Reciprocidad}

"Cuando el individuo considera que las retribuciones que obtiene de la organización son mayores que sus esfuerzos, percibe la relación como exitosa. Por el contrario, si no tiene esta percepción, se vuelve propenso a deteriorar su relación con la organización, y puede llegar incluso a abandonarla." Uría (2011). En todas las organizaciones deben los lograr satisfacer las expectativas mutuas, tanto el de la organización, como el del individuo.

Proceso de complementación en donde el individuo y la organización se vuelven parte uno del otro. Marín et al. (1992), menciona la reciprocidad como la "contribución de los diferentes individuos $y$ grupos formales $e$ informales, en el logro de objetivos". Y nos muestra los siguientes indicadores:

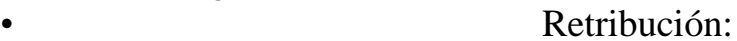

La organización tiene que optimizar los contenidos de remuneraciones, de las relaciones laborales, en beneficio de sus empleados, para contribuir a su realización personal y desarrollo social.

- $\quad$ Equidad:

Acceso a las remuneraciones por medio de un sistema equitativo que trate a todos en condiciones de igualdad.

\section{Participación}

Rebollo y Martí (2002) señalan que la participación no es una finalidad en sí misma sino 
un medio para conseguir algo, la participación debe ser comprendida como un derecho y no como un cumplimiento formal. Ambos autores sugieren debe entendérsele no únicamente como un derecho que tienen a reunirse, sino como un derecho a conformarse en grupos para lograr un objetivo común y ejercerse como un proceso que no se trate solamente para conformar al equipo, al contrario, debe entender para el logro, seguimiento y evaluación de objetivos. Es decir, contribuir en los diferentes grupos, para el logro de metas y objetivos.

Indicadores de participación

- Intercambio de información: Compartir la información fundamental entre los grupos para el desarrollo de objetivos y metas comunes.

\section{MÉTODO}

Para poder proponer una mejora en una maquiladora dedicada a la fabricación de microcircuitos, fue necesario construir un instrumento de medición, el cual pretende validar en qué dimensión del clima organizacional se presenta mayor influencia sobre la productividad dentro del área de finanzas. El instrumento se compone principalmente de cuatro dimensiones: liderazgo, motivación, participación y reciprocidad, mismas que integran el concepto de clima organizacional. La identificación de la dimensión de mayor influencia permitirá atender

Tabla 1 Operacionalización de las variables.

\begin{tabular}{|c|c|c|}
\hline Variable & Dimensiones & Indicador \\
\hline \multirow{9}{*}{$\begin{array}{c}\text { Clima } \\
\text { Organizaciona } \\
1\end{array}$} & \multirow{2}{*}{ Liderazgo } & Dirección \\
\hline & & Estimulo en equipo \\
\hline & \multirow{3}{*}{ Motivación } & $\begin{array}{l}\text { Adecuaciones de las } \\
\text { condiciones de trabajo }\end{array}$ \\
\hline & & Reconocimiento \\
\hline & & Realización personal \\
\hline & \multirow{2}{*}{ Reciprocidad } & Retribución \\
\hline & & Equidad \\
\hline & \multirow{2}{*}{ Participación } & Involucramiento en el cambio \\
\hline & & Intercambio de información \\
\hline
\end{tabular}

Fuente: elaboración propia.

Para la validación fue necesario aplicar una prueba piloto a catorce sujetos de estudio, los
- Involucramiento en el cambio: Adoptar una actitud y postura que se comprometiente al cambio.

Pastor (2018), en la justificación de su investigación hace hincapié a que si se desempeña una persona en un ambiente laboral favorable se desarrolla una satisfacción personal, por lo que menciona que es motivo fundamental para que las organizaciones reparen la percepción que tienen sus empleados para mejorar la productividad y ser más competitivos, por lo que su finalidad fue proporcionar bases para definir una nueva política laboral para los trabajadores de la empresa.

de manera eficiente las áreas de oportunidad en la empresa.

Las dimensiones desarrolladas para el clima organizacional en este trabajo se subdividieron en: liderazgo, motivación, reciprocidad y participación. Liderazgo cuenta con los indicadores de dirección y estimulo en equipo; Motivación se desplego en tres indicadores adecuaciones de las condiciones de trabajo, reconocimiento y realización personal; Participación con sus indicadores de involucramiento en el cambio e intercambio de información (Tabla 1).

cuales fueron seleccionados al azar de los tres departamentos que integran el área de finanzas, dando como resultado el $40 \%$ de la población a encuestar. El instrumento tipo encuesta consta de 29 ítems de escala Likert con cinco opciones como posibles respuestas. Para lograr la claridad de los reactivos y otorgarle el orden correcto se acudió a la revisión de expertos en el tema de finanzas. Para la fiabilidad y validez del instrumento se utilizó el programa SPSS, del cual se aplicaron las pruebas de alfa de cronbach, comunalidades, KMO y Bartlett's. 


\section{RESULTADOS}

De acuerdo con Oviedo y Campos-Arias (2005), la confiabilidad es el grado en que un instrumento mide una muestra de la población, la prueba de alfa de cronbach determina en sus resultados que el puntaje más cercano a 1 es el que presenta mayor consistencia mayor en el instrumento de medida. Según George y Mallery (2003) sugieren que los coeficientes de alfa de cronbach alcanzados en los análisis estadísticos se ubican en: $>0.9$ excelente; $>0.8$ bueno; $>0.7$ aceptable y; $>0.6$ cuestionable. Los resultados obtenidos en la validación de este instrumento muestran un alfa de cronbach para Liderazgo de .851; Motivación .712; Reciprocidad .822; y Participación con. 752 (Tabla 2).

\begin{tabular}{|c|c|c|c|}
\hline Dimensión & ítems & No. & $\begin{array}{c}\text { Alfa de } \\
\text { Cronbach }\end{array}$ \\
\hline \multirow{2}{*}{ Liderazgo } & LET1-LET3 & 3 & \multirow{2}{*}{.851} \\
\hline & LDR4-LDR8 & 5 & \\
\hline \multirow{3}{*}{ Motivación } & MCT10 & 1 & \multirow{3}{*}{.712} \\
\hline & MR12-MR14 & 2 & \\
\hline & MRP13-MRP17 & 3 & \\
\hline \multirow{2}{*}{ Reciprocidad } & RE18 & 1 & \multirow{2}{*}{.822} \\
\hline & RR19-RR23 & 4 & \\
\hline \multirow{2}{*}{ Participación } & PII24-PII25 & 2 & \multirow{2}{*}{.752} \\
\hline & PIC28-PIC29 & 2 & \\
\hline
\end{tabular}

Fuente: elaboración propia

La prueba KMO mide la adecuación que existe en la muestra, lo cual indica que tan apropiado es aplicar el análisis factorial, confirmando que los valores entre 0.05 y 1 apropiado aplicar dicho análisis. La prueba de esfericidad de Barlett comprueba las correlaciones y se conocen como validos aquellos resultados que resulten menores a 0.05. Montoya (2007). Los resultados obtenidos en las pruebas realizadas muestran un KMO y esfericidad de Barlett para Liderazgo de .592 y .000; motivación .558 y .023; Reciprocidad con

\section{CONCLUSIONES}

Esta investigación comprueba que la hipótesis planteada sobre la aplicación de un instrumento de medición que puede detectar que dimensión del clima organizacional influye más en el incremento de la productividad, es confiable y valido; siendo la dimensión de reciprocidad la que arrojo mejores índices en las pruebas
.713 y .005; y Participación con .544 y.002; lo que significa que cada una de las dimensiones cumple con las condiciones idóneas para realizar un análisis factorial y se comprueban las correlaciones entre las dimensiones (Tabla 3 ).

Tabla 3 Prueba KMO y esfericidad de Bartlett.

\begin{tabular}{|c|l|c|c|}
\hline Dimensión & \multicolumn{1}{|c|}{ Ítems } & \multirow{2}{*}{ KMO } & Barlett \\
\hline \multirow{2}{*}{ Liderazgo } & LET1-LET3 & \multirow{2}{*}{.592} & .000 \\
\cline { 2 - 2 } & LDR4-LDR8 & & \\
\hline \multirow{3}{*}{ Motivación } & MCT10 & \multirow{2}{*}{.558} & \\
\cline { 2 - 2 } & MR12-MR14 & .023 \\
\cline { 2 - 2 } & MRP13-MRP17 & & \\
\hline \multirow{2}{*}{ Reciprocidad } & RE18 & \multirow{2}{*}{.713} & .005 \\
\cline { 2 - 2 } & RR19-RR23 & & \\
\hline \multirow{2}{*}{ Participación } & PII24-PII25 & \multirow{2}{*}{.544} & .002 \\
\cline { 2 - 2 } & PIC28-PIC29 & & \\
\hline
\end{tabular}

Fuente: elaboración propia

De acuerdo con Detrinidad (2016), la comunalidad es el grado en que cada ítem se correlaciona con los otros ítems, y se considera que si el índice corresponde a un número mayor a 0.4 realmente exista tal correlación. Con la siguiente información se confirma que los datos obtenidos de las pruebas estadísticas realizadas cumplen con los criterios para la viabilidad del instrumento y conocer las correlaciones que existe entre los ítems. (Ver tabla 4).

Como instrumento final ya con las pruebas realizadas, se optó por eliminar 6 ítems que no contaban con los índices adecuados para su validación y fiabilidad quedando con un total de 23 preguntas, mismo que se puede observar en Anexo 1.

estadísticas realizadas con respecto a las otras. A su vez se cumplió con el objetivo planteado de validar el instrumento que permita identificar la dimensión con mayor influencia en la productividad. El instrumento validado se utilizará para la continuidad de la investigación que se encuentra en curso para posteriormente ser aplicado al total de población. 
El clima organizacional influye en la satisfacción y en el desempeño de los miembros de una organización. Este ejerce influencia directa en el comportamiento y en la conducta. Se indica que es la manera en que el colaborador percibe su productividad, sus rendimientos, su trabajo, y la satisfacción en las actividades que desempeña. Los trabajadores tendrán un mayor desenvolvimiento con mayor eficiencia y con actitud para desempeñarse mejor si se tiene un clima favorable por lo que este instrumento es relevante para el estudio de las ciencias sociales ya que proporciona información valida que puede mejorar cualquier organización, sin ser específicamente del sector maquiladora. 


\section{REFERENCIAS}

Cubas, N. (2016). La motivación y su influencia en el rendimiento laboral del personal en la LE-ADE DEPORTIVO SAC - CHICLAYO. [Tesis de Licenciatura, Universidad Señor de Sipán]. http://repositorio.uss.edu.pe/bitstream/handle/uss/4366/Cubas\%20Medina.pdf?sequence=1\&isAllo wed $=\mathrm{y}$

Detrinidad, E. (2016). Análisis factorial exploratorio y confirmatorio aplicado al modelo de secularización propuestos por Inglegart-Norris. Periodo 2010-2014 (Estudio de caso España, Estados Unidos Alemana, Holanda) WSV. [Tesis de maestría, Universidad de Granada]. https://masteres.ugr.es/moea/pages/curso201516/tfm1516/detrinidad barquero tfm/!

George, D., \& Mallery, P. (2003). SPSS for Windows step by step: A simple guide and reference. 11.0 update (4thed.). Boston: Allyn \& Bacon.

Lussier y Achua. (Eds.). (2011). Liderazgo, teoria,aplicacion y desarollo de habilidades. Mexico DF.: Cuarta Edicion, Cengage Learning.

Luthans F. (2008). Comportamiento Organizacional. México: McGraw - Hill.

Marín, J. M., Melgar, A., y Castaño, C. (1992). Teorıa y Tecnicas de Desarrollo Organizacional, Guatemala, ' Guatemala: Organizacion Panamericana de la Salud, PSDCG-T.10, Volumen III.

Martínez, R. y Ramírez M. (2010). La motivación y el clima organizacional en la Facultad de Contaduría Pública de la Benemérita Universidad Autónoma de Puebla. Revista Internacional de Administración y Finanzas, 3(1), 39-48.

Montoya, O. (2007). Aplicación del análisis factorial a la investigación de mercados. Caso de estudio. https://www.redalyc.org/pdf/849/84903549.pdf

Oviedo, H. y Campos-Arias A. (2005). Aproximación al uso del coeficiente alfa de Cronbach. Revista colombiana de psiquiatría vol. XXXIV no. 4. https://www.redalyc.org/pdf/806/80634409.pd

Pastor, A. (2018). Clima organizacional y desempeño en trabajadores administrativos de una empresa privada de combustibles e hidrocarburos, Lima 2017. [Tesis de licenciatura, Universidad San Ignacio de Loyola].

Rebollo, O. y Marti, J. (2002). Eines per la participación ciutadana: bases, mètodes i tècniques. OrganismeAutònom Flor de Maig, Diputació de Barcelona. En Martí, J., Pascual, J. y Rebollo, O. (coords). Participación y desarrollo comunitario en medio urbano. Experiencias y reflexiones. Construyendo ciudadanía / 7. España: IEPALA Editorial / CIMAS, 2005.

Recio, R, Balderas, M. y Martinez, E. (2016). Clima organzacional y satisfaccion laboral en trabajadores de escuelas de educacion basica de Ciudad Fernandez, S.L.P. https://sices.guanajuato.gob.mx/resources/ideas/ebooks/144/descargas.pdf

Salazar, J., Guerrero, J., Machado, Y. y, Cañedo, R. (2009). Clima y cultura organizacional: dos componentes esenciales en la productividad laboral. ACIMED, 20(4), 67-75.

Segredo, A. (2013). Clima organizacional en la gestión del cambio para el desarrollo de la organización. Revista Cubana de Salud Pública, 39(2), 385-393.

Sum, M. (2015). Motivación y desempeño laboral. [Tesis de licenciatura, Universidad Rafael Landívar].

Uría, D. (2011). El clima organizacional y su incidencia en el desempeño laboral de los trabajadores de Andelas Cía. LTDA. de la ciudad de Ambato. [Tesis de licenciatura, Universidad Técnica de Ambato]. https://repositorio.uta.edu.ec/handle/123456789/1376 


\title{
ANEXOS
}

\section{Anexo 1}

\author{
Universidad Autónoma de Baja California \\ Facultad de Ciencias Administrativas \\ Maestría en Administración
}

Tema: Influencia del clima organizacional en el desempeño laboral del área de finanzas de una maquiladora en Mexicali, Baja California.

|

La presente encuesta es parte de una investigación que tiene por finalidad evaluar la influencia que existe entre el clima organizacional y el desempeño laboral. La encuesta es confidencial y anónima, por lo cual se agradece ser lo más sincero posible.

Género: $\mathrm{H} / \mathrm{M}$

\section{Antigüedad en la empresa:}

Departamento:

Edad:

Instrucciones: Lea detenidamente cada pregunta y marque con una $\mathrm{X}$ su respuesta.

\begin{tabular}{|c|c|c|c|c|c|c|}
\hline No. & Pregunta & Siempre & $\begin{array}{c}\text { Casi } \\
\text { Siempre }\end{array}$ & $\begin{array}{l}\text { Algunas } \\
\text { Veces }\end{array}$ & $\begin{array}{l}\text { Muy pocas } \\
\text { veces }\end{array}$ & Nunca \\
\hline \multicolumn{7}{|c|}{ Liderazgo } \\
\hline 1. & $\begin{array}{l}\text { Mi lider se preocupa por mantener un buen } \\
\text { ambiente de trabajo. }\end{array}$ & & & & & \\
\hline 2. & $\begin{array}{l}\text { Mi lider fomenta las relaciones humanas con } \\
\text { el personal. }\end{array}$ & & & & & \\
\hline 3. & $\begin{array}{l}\text { Mi lider acepta con frecuencia las ideas que } \\
\text { aporto para mejorar la calidad del trabajo. }\end{array}$ & & & & & \\
\hline 4. & $\begin{array}{l}\text { Recibo ayuda por parte de mi lider cuando } \\
\text { se presenta un problema. }\end{array}$ & & & & & \\
\hline 5. & $\begin{array}{l}\text { Recibo retroalimentación por parte de mi } \\
\text { líder sobre mi desempeño. }\end{array}$ & & & & & \\
\hline 6. & $\begin{array}{l}\text { Mi lider asigna las actividades de manera } \\
\text { efectiva. }\end{array}$ & & & & & \\
\hline 7. & $\begin{array}{l}\text { Puedo hablar libremente con mi líder cuando } \\
\text { estoy en desacuerdo sobre el trabajo que nos } \\
\text { asigna. }\end{array}$ & & & & & \\
\hline 8. & $\begin{array}{l}\text { Cuando llego a cometer algún error, mi líder } \\
\text { lo detecta oportunamente y me informa de } \\
\text { manera adecuada. }\end{array}$ & & & & & \\
\hline \multicolumn{7}{|c|}{ Motivación } \\
\hline 10 & $\begin{array}{l}\text { Cuento con las herramientas necesarias para } \\
\text { desempeñar mi trabajo de manera efectiva. } \\
\text { (Computadora, mouse etc.) }\end{array}$ & & & & & \\
\hline 12. & $\begin{array}{l}\text { He tenido oportunidades de crecimiento } \\
\text { laboral }\end{array}$ & & & & & \\
\hline 13. & $\begin{array}{l}\text { En este trabajo me siento realizado } \\
\text { profesionalmente. }\end{array}$ & & & & & \\
\hline 14. & $\begin{array}{l}\text { La empresa estimula a su personal y } \\
\text { reconoce los esfuerzos adicionales }\end{array}$ & & & & & \\
\hline 15. & $\begin{array}{l}\text { Me siento motivado de formar parte del área } \\
\text { de finanzas }\end{array}$ & & & & & \\
\hline
\end{tabular}




\section{Universidad Autónoma de Baja California \\ Facultad de Ciencias Administrativas \\ Maestría en Administración}

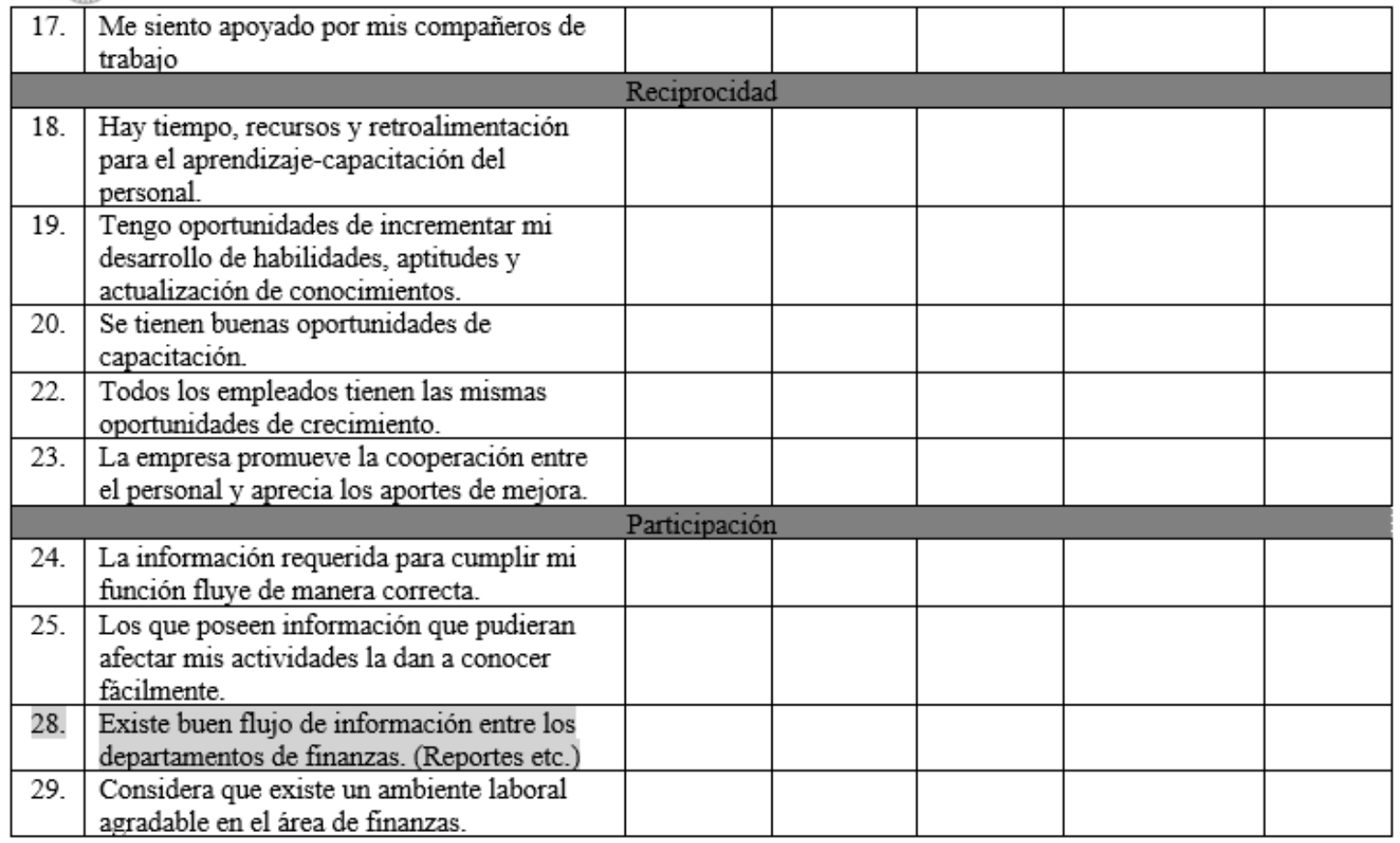

Gracias por su participación 